\title{
A Design of Fuzzy-PID Controller of Constant Temperature for Semiconductor Laser Diodes
}

\author{
Ji Zou ${ }^{1}$, Chao Zhang ${ }^{1}$ and Duan-yuan Bai $^{2}$ \\ 1Changchun University, Changchun, China \\ 2Changchun University of Science and Technology, Changchun, China \\ bdybai@hotmail.com
}

\begin{abstract}
The paper introduces two types of precise temperature controllers for high power semiconductor laser diodes. We apply a category of self-tuning parameter fuzzy-PID controller and a kind of neuronal network optimized rules fuzzy-PID controller to realize stable temperature controlling. Also we simulate this two methods using Matlab. The result proves that the new method has more superiority than traditional means, such as PID controlling, etc. And further improvement on constant temperature control will inevitably promote the reliability of $L D$.
\end{abstract}

Keywords: Laser-Diode of Semiconductor, Fuzzy-PID Controller, Constant Temperature

\section{Introduction}

High power semiconductor lasers are widely used in the fields of communication, industry, medicine, military affairs and technical aeronautics, for its excellences, such as small size, long life-time, high efficiency, fast response, etc. Semiconductor lasers cannot convert electric power to optical power entirely. In the process of carrier irradiation recombination into photons, non-radiation and free-carrier absorption transforming electric power into heat cause device temperature rising. Semiconductor laser-diodes are sensitive to temperature, if the temperature goes too high it will inevitably bring the threshold current increasing, emission wavelength red-shift, more inferior characteristics, so the device reliability becomes disappointing. Thus, the control of device temperature is especially important. This paper designs a type of fuzzy-PID controller and a kind of controller by fuzzy-rules is optimized with neuronal network; the fuzzy system also endure human illation ability to neuronal network, combine neuronal network with fuzzyPID control method will obtain a new temperature control system with better robustness, rapid response, stable precision, and then promote the reliability of high power semiconductor laser diodes.

\section{Design of Fuzzy- PID Controller}

For laser diodes, especially high power laser diodes and its arrays which needs high stability, we use the TEC as the performance piece for temperature control system. Changing the direction of the current flow will realize refrigeration or heating, regulating the voltage will obtain accurate temperature control.

For accurate temperature control, we use fuzzy-PID method, and use simulink in matlab to simulate the process. 


\subsection{Mathematical Model of Temperature Control System}

In accordance with the data of TEC thermal characteristics in reference [1], we will easily obtain the temperature data. Considering the thermal inertia of thermistor and the TEC, we get the transfer function of temperature and voltage like formula (1):

$$
G(s)=\frac{T(s)}{U(s)}=\frac{2.1}{52 s+1} e^{-2.3 s}
$$

\subsection{Design of Fuzzy-PID Controller}

Since system contains the time-delay we introduce Fuzzy rules to the PID controller to get better controlling quality. The structure of the system is show in Figure 1. Figure 2 shows the specific constitute of the subsystem.

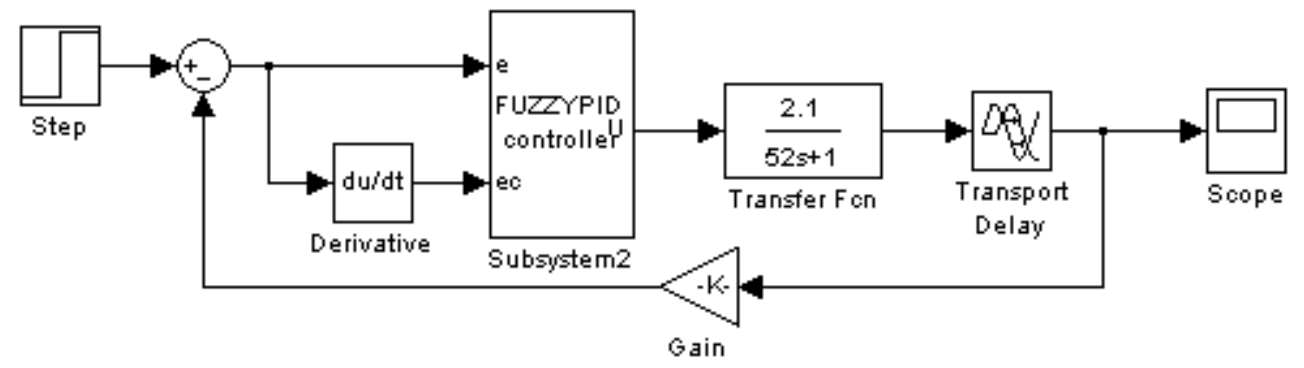

Figure 1. The Structure of Fuzzy-PID Controlled LD Temperature Controlling System

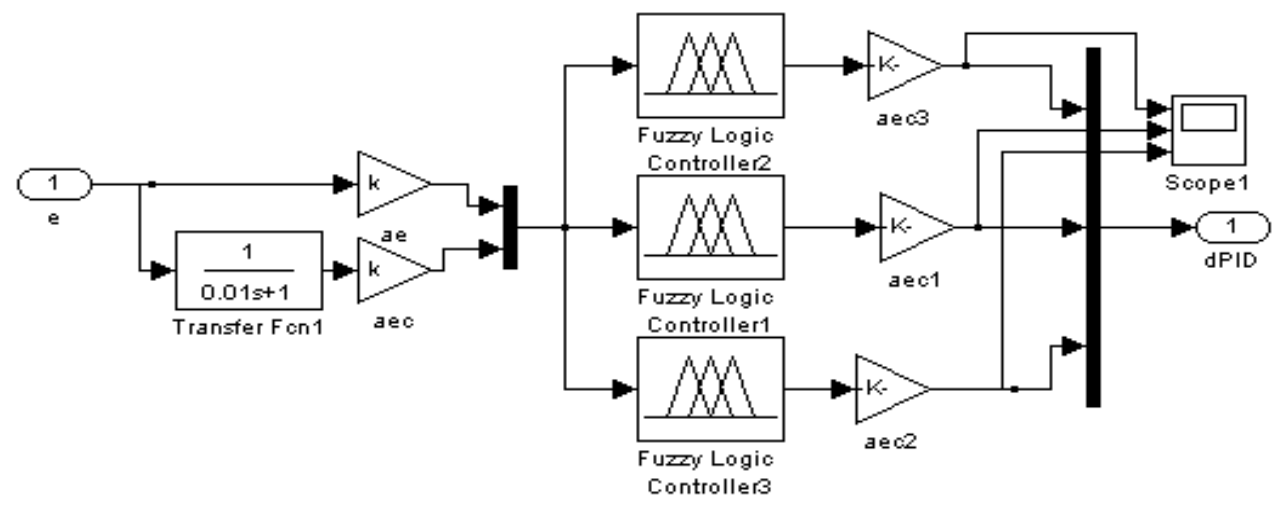

Figure 2. The Constitute of the Subsystem

\subsection{Determination of Fuzzy Control Rule Sets}

The fuzzy rules of $\Delta K p$ are determined like Table 1. For example: when controlled variable overshoot of temperature or temperature difference becomes larger, thus overshoot increases rapidly, we take measures to amplify the impact of proportionality factor. 
Table 1. The Fuzzy-rule of $\Delta K p$

\begin{tabular}{|c|c|c|c|c|c|c|c|c|}
\hline \multicolumn{2}{|c|}{} & \multicolumn{7}{|c|}{$\mathrm{E}$} \\
\cline { 2 - 9 } \multicolumn{2}{|c|}{$\Delta K_{p}$} & NB & NM & NS & ZO & PS & PM & PB \\
\hline \multirow{4}{*}{ EC NB } & PB & PM & PM & NB & NM & Z0 & ZO \\
\cline { 2 - 9 } & NM & PB & PM & PS & NM & NS & ZO & ZO \\
\cline { 2 - 9 } & NS & PB & PS & ZO & ZO & PS & PS & PB \\
\cline { 2 - 9 } & ZO & PB & PS & ZO & ZO & ZO & PM & PB \\
\cline { 2 - 9 } & PS & PM & PS & PS & ZO & PM & PM & PB \\
\cline { 2 - 9 } & PM & PS & ZO & NS & PS & PS & PM & PB \\
\cline { 2 - 9 } & PB & PS & ZO & NM & PS & PM & PB & PB \\
\hline
\end{tabular}

The fuzzy rules of $\Delta K i$ are determined like table 2. For example: when negative deviation is very large, deviation rate is positive and big, and the rate of deviation decreases rapidly, we can keep the $\Delta K i$ remain the same, etc.

Table 2. The Fuzzy-rule of $\Delta K i$

\begin{tabular}{|c|c|c|c|c|c|c|c|c|}
\hline \multicolumn{2}{|c|}{} & \multicolumn{7}{|c|}{$\mathrm{E}$} \\
\cline { 2 - 9 } \multicolumn{2}{|c|}{$\Delta K_{i}$} & NB & NM & NS & ZO & PS & PM & PB \\
\hline \multirow{4}{*}{ EC } & NB & PB & PB & PB & NM & NM & ZO & ZO \\
\cline { 2 - 9 } & NM & PB & PM & PM & NS & NS & ZO & PS \\
\cline { 2 - 9 } & NS & PB & PB & PM & ZO & ZO & PS & PB \\
\cline { 2 - 9 } & ZO & PM & PM & PS & ZO & PS & PM & PB \\
\cline { 2 - 9 } & PS & PM & PS & ZO & PS & PM & PM & PB \\
\cline { 2 - 9 } & PM & PS & ZO & NS & NS & PM & PB & PB \\
\cline { 2 - 9 } & PB & ZO & NM & NM & NM & PB & PB & PB \\
\hline
\end{tabular}

The fuzzy rules of $\Delta K d$ are determined like Table 3. For example: if the deviation at the most value in a reverse direction, and changing rate also like deviation, and controlled variable is much smaller than the steady-state value and continue to decrease rapidly, then we should enhance the effect of differential, etc.

Table 3. The Fuzzy-rule of $\Delta K d$

\begin{tabular}{|c|c|c|c|c|c|c|c|c|}
\hline \multicolumn{2}{|c|}{} & \multicolumn{7}{|c|}{$\mathrm{E}$} \\
\cline { 2 - 9 } \multicolumn{2}{|c|}{$\Delta K_{d}$} & NB & NM & NS & ZO & PS & PM & PB \\
\hline \multirow{7}{*}{ EC } & NB & PB & PM & PB & PB & PB & ZO & NB \\
\cline { 2 - 9 } & NM & PM & PS & PM & PM & PM & NS & NB \\
\cline { 2 - 9 } & NS & PS & ZO & PS & PS & PS & NM & NB \\
\cline { 2 - 9 } & ZO & NB & ZO & PS & PS & PS & ZO & PS \\
\cline { 2 - 9 } & PS & NB & NB & PS & PS & PS & ZO & PS \\
\cline { 2 - 9 } & PM & NB & NS & PM & PM & PM & PS & PM \\
\hline
\end{tabular}




\begin{tabular}{|l|l|l|l|l|l|l|l|l|}
\hline & PB & NB & ZO & PB & PB & PB & PM & PB \\
\hline
\end{tabular}

\subsection{Simulation Result}

The simulation result by Fuzzy-PID control method and PID control method is shown in Figure 3. From the figure, we can easily find that the method of fuzzy-PID is superior than that of PID because the result of fuzzy-PID method has faster rise-time and lower overshoot.

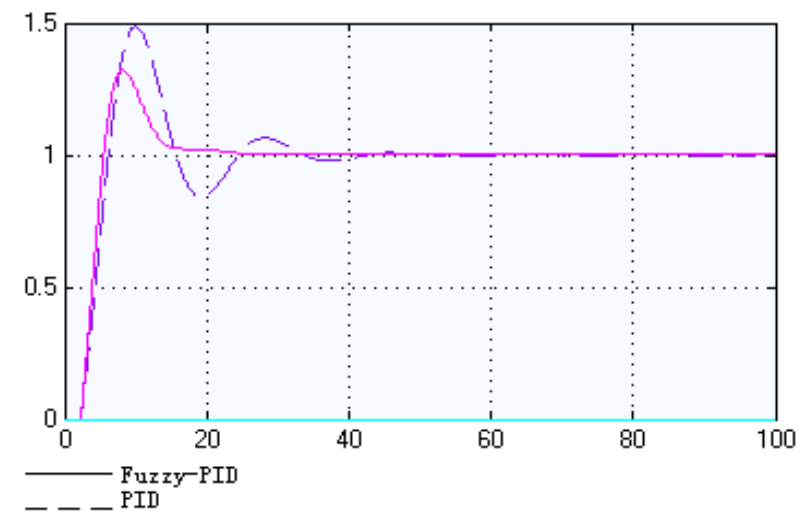

\section{Figure 3. The Comparison for the Simulation Result of Fuzzy-PID Control and PID Control}

\subsection{The Optimized Fuzzy-rules with Neuronal Network}

We using neuronal network to optimize the fuzzy-rules to get better controlling effect. This time we rebuild the fuzzy-controller with Sugeno type. All its functions for grade of membership and fuzzy controlling rules are all obtained by neuronal network training.

\subsection{Neuronal Network Optimized Fuzzy-rules}

The training process is like this: first, we should get the expecting data of the output wave, and this is tightly associated with the best temperature changing wave. For this we use an ideal step response data of TEC temperature adjusting data to training. For example: if in the practice use, we need the step response of the TEC temperature control system like Figure 4, then we extract the data show in Table 4.

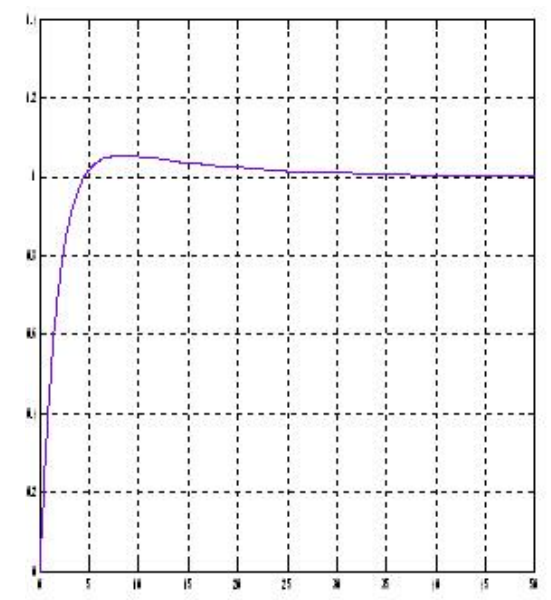

Figure 4. Expected Step Response 
Table 4. The Data of Expected Step Response

\begin{tabular}{|c|c|}
\hline 0 & 0 \\
\hline 0 & 0 \\
\hline $2.10272 \mathrm{E}-06$ & $1.04472 \mathrm{E}-06$ \\
\hline $3.92682 \mathrm{E}-06$ & $2.13685 \mathrm{E}-06$ \\
\hline $6.16191 \mathrm{E}-06$ & $3.52173 \mathrm{E}-06$ \\
\hline $9.77199 \mathrm{E}-06$ & $5.77403 \mathrm{E}-06$ \\
\hline $8.29736 \mathrm{E}-05$ & $5.15207 \mathrm{E}-05$ \\
\hline 0.000233365 & 0.000145504 \\
\hline 0.000745631 & 0.00046556 \\
\hline 0.00246804 & 0.001540839 \\
\hline$\ldots$ & $\ldots \ldots \ldots \ldots$ \\
\hline 49.9576896 & 1.001585097 \\
\hline 49.9676896 & 1.001583685 \\
\hline 49.9776896 & 1.001582273 \\
\hline 49.9876896 & 1.001580863 \\
\hline 49.9976896 & 1.001579455 \\
\hline 50 & 1.001579129 \\
\hline
\end{tabular}

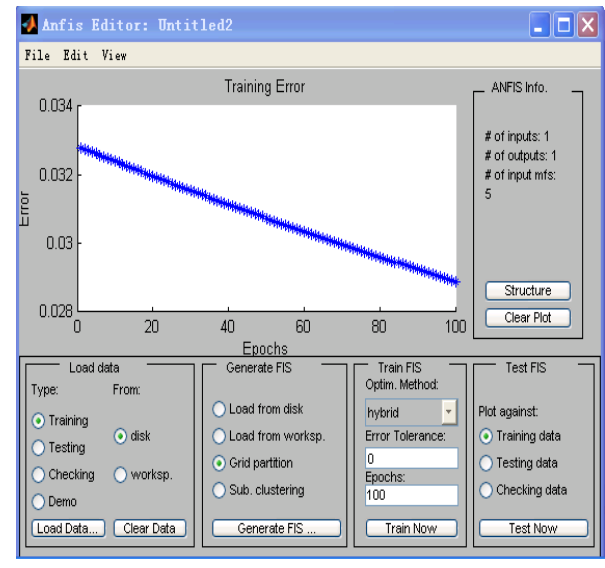

Figure 5. The Training Error

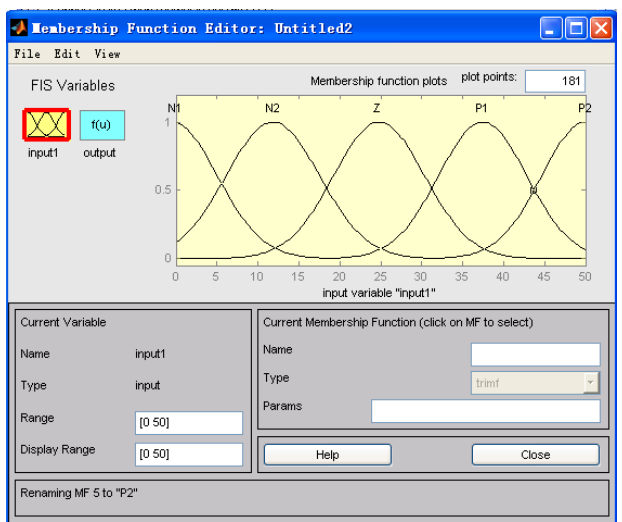

Figure 7. The Obtained Functions

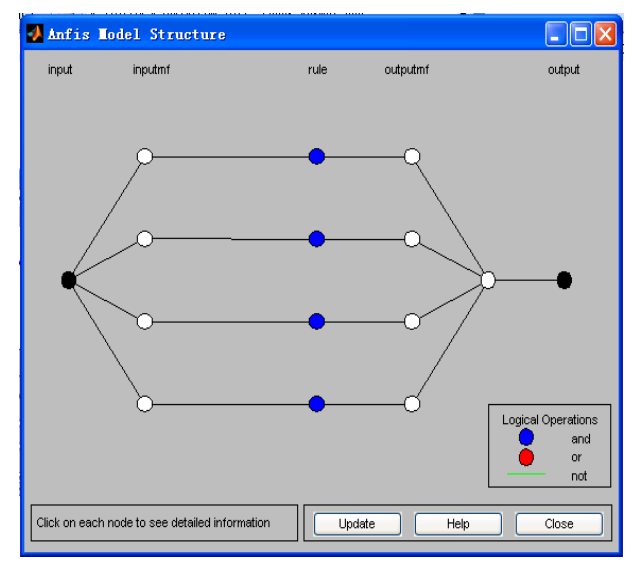

Figure 6. The Obtained Structure

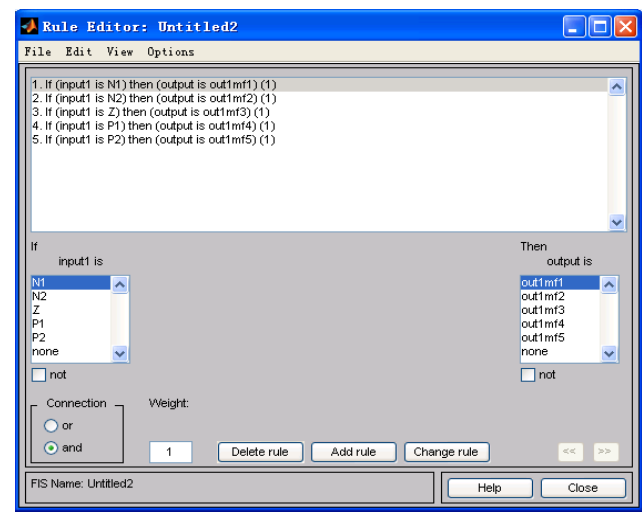

Figure 8. Fuzzy Rules of Membership

After training, we get the error is 0.029 , the obtained functions for grade of membership are(2)-(6). Here, we used Gaussian function:

$$
N 2: f_{1}(x)=e^{-\frac{(x+0.4533)^{2}}{60.808}}
$$




$$
\begin{aligned}
& N 1: f_{2}(x)=e^{-\frac{(x-11.83)^{2}}{65.4368}} \\
& Z: f_{3}(x)=e^{-\frac{(x-24.8)^{2}}{61.96072}} \\
& P 1: f_{4}(x)=e^{-\frac{(x-37.45)^{2}}{58.23632}} \\
& P 2: f_{5}(x)=e^{-\frac{(x-49.98)^{2}}{56.8818}}
\end{aligned}
$$

And the obtained optimized fuzzy-rule are as follows:

$$
\begin{aligned}
& \mathrm{R} 1: \text { if } T \text { is } N 2 \text { then } U \text { is } U 1=0.2767 x+0.2355 \\
& \mathrm{R} 2: \text { if } T \text { is } N 1 \text { then } U \text { is } U 1=0.07078 x-0.008183 \\
& \mathrm{R} 3: \text { if } T \text { is } Z \text { then } U \text { is } U 1=0.2791 x+0.2663 \\
& \mathrm{R} 4: \text { if } T \text { is } P 1 \text { then } U \text { is } U 1=0.01576 x+0.3972 \\
& \mathrm{R} 5: \text { if } T \text { is } P 2 \text { then } U \text { is } U 1=0.1343 x+0.3302
\end{aligned}
$$

\subsection{Simulation Result}

Comparing Figure 9 with Figure 3, the optimized fuzzy-rules improve the overshoot, obtain a swift rising time, and the response relatively stable. When comparing with aiding effect of Figure 4, though the incremental of the optimized fuzzy-rules can adjust the control effect, still do not solve the delay time problem completely.

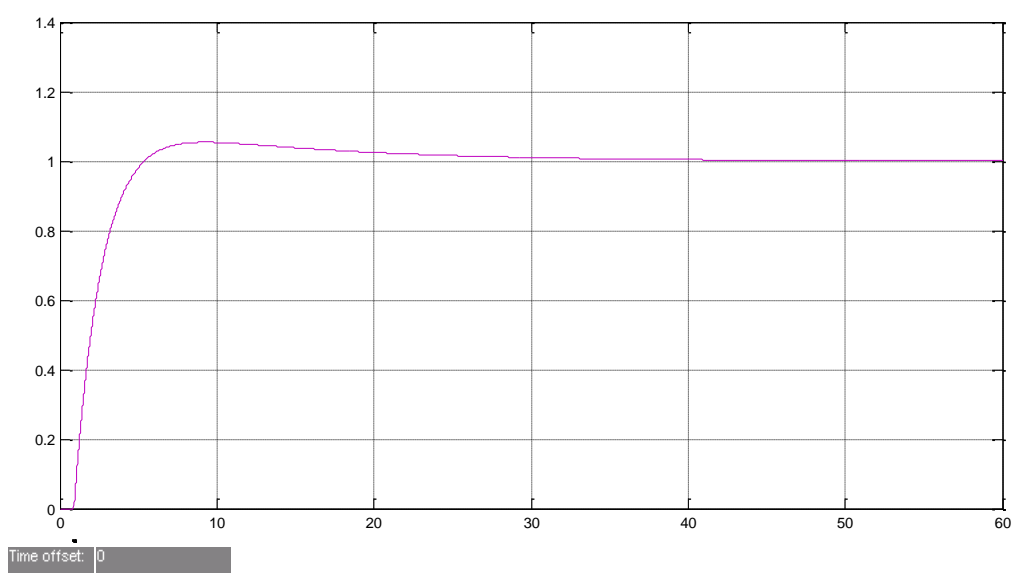

Figure 9. The Simulation Result using Neuronal Network Optimized Fuzzyrules

\section{Summary}

This work focused on two type of fuzzy-PID constant temperature controller for high power semiconductor laser diodes with TEC. The simulation shows the satisfactory results. The methods can effectively attain stable temperature control for $\mathrm{LD}$, which have valuable guidance and practical importance for the development of $L D$ temperature control research. And the improvement of constant temperature control will inevitably promote the reliability of $\mathrm{LD}$. 


\section{References}

[1]. L. F. Sun, "The Design of Driver on Switch-mode Constant Temperature Control System of Semiconductor Laser Diode", Master Dissertation, (2004).

[2]. X. M. Shi, "Fuzzy Control and Its MATLAB Simulation", Tsinghua University Publishing House, (2010).

[3]. S. P. Ai, "The Research of Control Mode and Programming on Constant Temperature Control Unit of Semiconductor Laser Diode", Master Dissertation, (2005).

[4]. W. Zhong, "Optimization Design for Auto Temperature Control System of Semiconductor Laser Diode", Journal of Anhui Vocational College of electronics and information Technology General, (2010).

[5]. S. P. Luan, "Dynamic Model Identification and Temperature Control Of a Themoelectric Cooler", Measurement Technology, (2010). 
International Journal of Control and Automation Vol. 8, No. 2 (2015) 